\title{
Offset Drift Dependence of Hall Cells with their Designed Geometry
}

\author{
Maria-Alexandra Paun, Jean-Michel Sallese and Maher Kayal
}

\begin{abstract}
In this paper, the performance of CMOS Hall Effect Sensors with four different geometries has been experimentally studied. Using a characteristic measurement system, the cells residual offset and its temperature behavior were determined. The offset, offset drift and sensitivity are quantities that were computed to determine the sensors performance. The temperature coefficient of specific parameters such as individual, residual offset and resistance has been also investigated. Therefore the optimum cell to fit the best in the performance specifications was identified. The variety of tested shapes ensures a good analysis on how the sensors performance changes with geometry.
\end{abstract}

Keywords - hall effect sensor, individual and residual offset drift, temperature coefficient

\section{INTRODUCTION}

$\mathbf{O}$ NE of the most widely used sensing technologies today consists of Hall effect sensors, based on magnetic phenomena. Many low-power applications like current sensing, position detection and contactless switching within automotive and industrial electronics use this kind of sensors [1], [2].

The sensitivity, offset and its temperature drift are important figures of merit in Hall sensors performance evaluation. There is a strong connection between the geometry and the Hall effect sensors performance as was studied by the authors in [3]. In order to predict and evaluate the sensors performance, three-dimensional physical simulations have been realized in order to facilitate the design process [4], [5] and automated measurements systems have been developed [6].

Several of Hall effect sensors were simulated and evaluated for numerical offset, drift, Hall voltage and sensitivity using three-dimensional physical simulations in a recent paper by the authors [4].

The present paper analyzes the influence of the shape, dimensions, on the Hall effect sensors performance, including sensitivity, offset and drift for optimal design of Hall effect sensors. In this sense, different Hall effect sensors were integrated in a $0.35 \mu \mathrm{m}$ CMOS technology. The diversity of the shapes and dimensions analyzed allows us to have a wide range of Hall cells amongst which to choose the one displaying the best performance for the project requirements. This is also a good opportunity to characterize the effect of temperature on individual, residual offset and resistance.

Section II presents the qualitative reasoning behind choosing the specific Hall effect sensors geometries and the design parameters for all proposed cells. The results corresponding to

M. A. Paun, J.-M. Sallese and M. Kayal are with the STI-IELElectronics Lab of Ecole Polytechnique Fédérale de Lausanne (EPFL) CH-1015 Lausanne, Switzerland (e-mails: maria-alexandra.paun@epfl.ch; jean-michel.sallese@epfl.ch; maher.kayal@epfl.ch). the magnetic equivalent residual offset and its behavior with the temperature are presented in Section III, with a comparative analysis on different Hall cell types. Experimental results for single and 4-phase residual offset voltage are also obtained for various Hall cells.

The temperature coefficient of the individual offset, residual offset (using 2-phase and 4-phase current spinning technique) and resistance has been computed for several cells. This section concludes which of the integrated shapes exhibited the best performance. The various shapes analyzed offer an overview on how the performance is related to the geometry. The end of Section III presents three-dimensional physical simulations for Hall cells performance investigation.

\section{Methodology}

\section{A. Hall Cells Integration}

Different Hall cells were integrated in a $0.35 \mu \mathrm{m}$ CMOS technology. All these cells are symmetric and orthogonal structures due to the fact that any geometrical mismatch could significantly increase the offset.

\section{B. The Chosen Structures}

The classical Greek cross used for this type of Hall cells with progressive increase in cell dimensions (basic, $\mathrm{L}$ and $\mathrm{XL}$ cells) and borderless cell (with small contacts situated far away from the $\mathrm{p}-\mathrm{n}$ junction) were integrated and analyzed.

\section{The Qualitative Reasoning behind the Shapes Choice}

The Hall cells are manufactured in a CMOS process and they basically have a p-substrate with a n-doped active region. On top they have a $p+$ diffusion layer to reduce the noise and prevent the current to flow under the contacts.

There is reasoning behind the choice of the four different Hall cells. The basic cell is taken as a reference, but it might suffer from a difference in the piezo-resistance due to the orientation of the axes. For the L and XL cells, the errors on the contour are less due to an averaging on a bigger size. The borderless shape might minimize the influence of any errors that could appear on the borders but the sensitivity is as well affected. The four tested Hall geometries (basic, L, XL and borderless cells) are presented in Fig. 1.

The design details are presented in Tab. I, together with measurements of the resistance, absolute sensitivity, offset drift (with four-phase current spinning technique). For the cross Hall cells, the geometrical correction factor $G$ was computed according to the formula in [7]. 

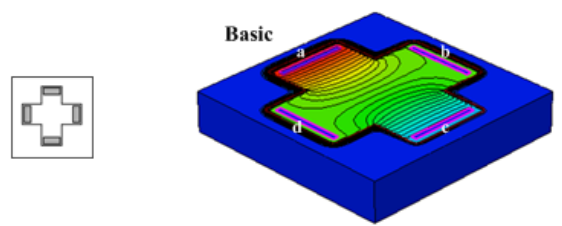

(a) Basic cell

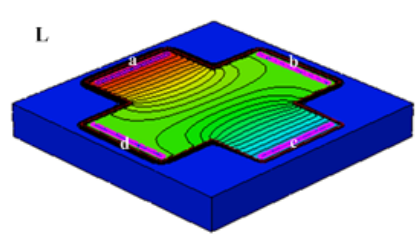

(b) L cell

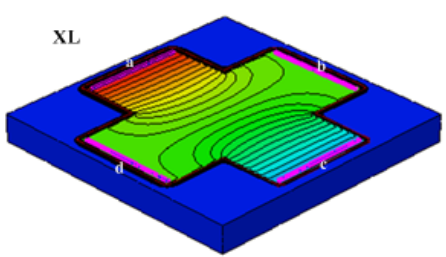

(c) XL cell

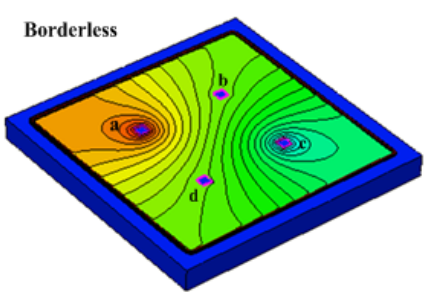

(d) Borderless cell

Fig. 1. The four analyzed Hall cells (2D and 3D simulated structures)

TABLE I

Design PARAMETERS OF THE HALl CELls

\begin{tabular}{|c|c|c|c|c|}
\hline Hall Cell & Basic & $\mathrm{L}$ & XL & Borderless \\
\hline $\begin{array}{c}R_{0}(k \Omega) @ T= \\
300 K, B=0 T\end{array}$ & 2.3 & 2.2 & 2.2 & 1.3 \\
\hline $\begin{array}{c}S_{A}(V / T) @ I_{\text {bias }}= \\
1 m A\end{array}$ & 0.082 & 0.082 & 0.082 & 0.032 \\
\hline Offset drift $\left(\mu T /{ }^{\circ} C\right)$ & 3.2 & 0.2 & 0.1 & 0.6 \\
\hline $\mathrm{L}(\mu m)$ & 21.6 & 32.4 & 43.2 & 50 \\
\hline $\mathrm{W}(\mu m)$ & 9.5 & 14.25 & 19 & 50 \\
\hline $\mathrm{L} / \mathrm{W}$ & 2.27 & 2.27 & 2.27 & 1 \\
\hline $\mathrm{s}(\mu m)$ & 8.8 & 13.55 & 18.3 & 2.3 \\
\hline $\mathrm{G}$ & 0.86 & 0.86 & 0.86 & - \\
\hline
\end{tabular}

$L$ and $W$ represent the cell length and width, respectively, of the active $\mathrm{N}$-well region while $s$ stands for contact length. The width of the contacts is in general imposed by the technology used in the Hall effect sensors fabrication process. The position of contacts with respect to borders is important in the offset analysis as contour errors might increase it.

Dimensions, via the geometrical correction factor as analyzed by authors in a recent paper [3], and distance between the contacts and the active region borders are important in the evaluation of the cells offset and sensitivity.

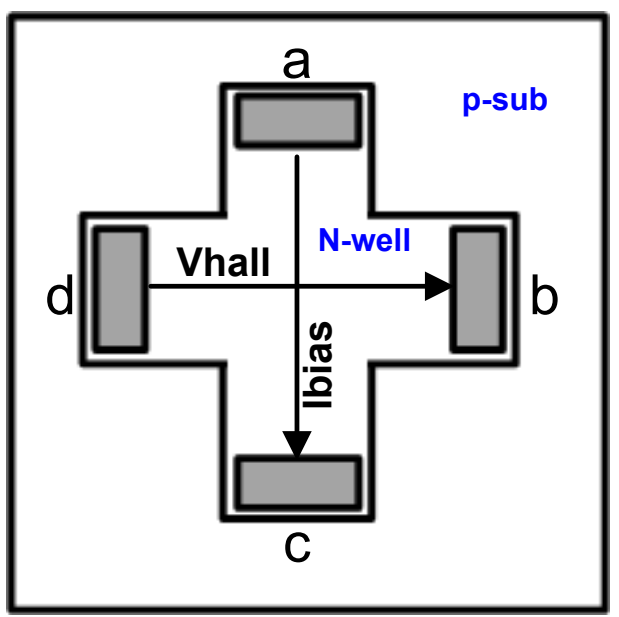

Fig. 2. Polarization of a Hall cell.

TABLE II

The Four Phases of the CurRent Spinning Technique

\begin{tabular}{|c|c|c|}
\hline Phases & $I_{\text {bias }}$ & $V_{\text {hall }}$ \\
\hline Phase 1 & a to c & b to d \\
\hline Phase 2 & d to b & a to c \\
\hline Phase 3 & c to a & d to b \\
\hline Phase 4 & b to d & c to a \\
\hline
\end{tabular}

Each structure is equipped with four contacts (denoted by $\mathrm{a}, \mathrm{b}, \mathrm{c}, \mathrm{d})$, among which two are for biasing the device and the other two opposite ones for measurement purposes, by collecting the voltage drop, as seen in Fig. 1. The four phases of the current spinning technique are presented in Tab. II.

The absolute sensitivity of Hall sensor is given by the ratio of the Hall voltage $\left(V_{H A L L}\right)$ to the magnetic field induction (B), as in the relation:

$$
S_{A}=\frac{V_{H A L L}}{B}=\frac{G r_{H}}{n q t} I_{b i a s}
$$

where $G$ is the geometrical correction factor, $I_{\text {bias }}$ is the biasing current, $r_{H}$ is the scattering factor of Silicon, usually $1.15, n$ is the carrier density and $t$ is the thickness of the active region [6].

\section{RESUlts AND Discussion}

All the Hall cells were designed for targeting specific objectives, namely the offset at $T=300 K$ less than $\pm 30 \mu T$ and the offset drift less than $\pm 0.3 \mu \mathrm{T} /{ }^{\circ} \mathrm{C}$. Our aim was to see which of the shapes fits the best within the specification interval and also has a behavior consistency. To this purpose, the measurements were performed on several samples.

Previous tests were performed on all the Hall cells, using an automated measurement system, presented in details by the authors in [6]. The advantage of the fully automatic system is the reliability, and the possibility to test all the cells, at the same time, under the same conditions. In this case the information on the residual offset was directly obtained.

In the present work, the structures were surrounded by the specific electronics and subsequently electrically tested 


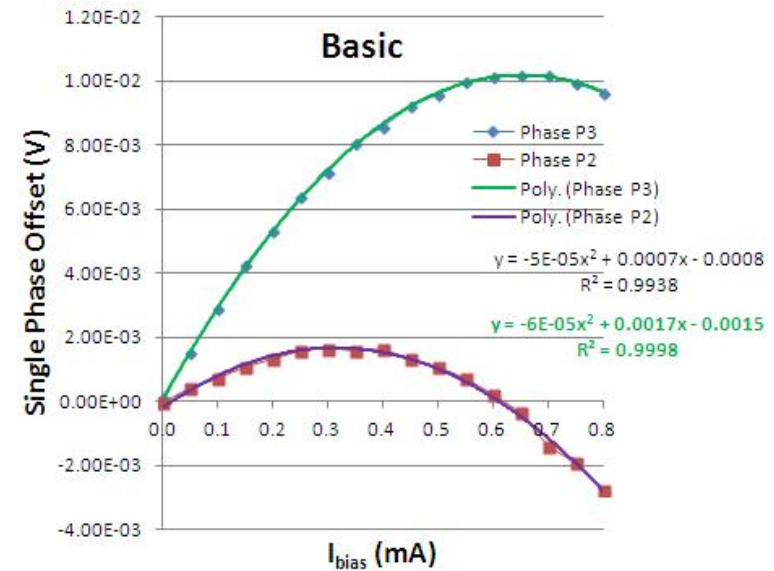

Fig. 3. Single phase offset (V) vs. $I_{\text {bias }}$ for basic cell.

but the phases were manually switched. In this way, we had information on each individual phase offset. 2-phase and 4phase spinning current could be analyzed, but some accuracy can be lost. In this way, information on their circuit behavior was obtained. A current biasing $(0-1 m A)$ was used for the Hall cells polarization. Measurements in the absence and presence of the magnetic field were performed. The offset measurements were performed in the absence of magnetic field while for Hall voltage and sensitivity estimations, the magnetic induction was $B=0.5 T$. The Hall cells offset was evaluated at room temperature and for certain temperatures in the interval $-40^{\circ}-125^{\circ} \mathrm{C}$. By accessing the latter information we extracted the offset drift, which is incorporated in Tab. I, after a 4-phase spinning of the sensors.

\section{A. Measurements of the Single Phase Offset}

The Hall voltage is affected by the offset through the relation:

$$
V_{\text {out }}=V_{H A L L}(B)+V_{\text {offset }}
$$

Even though the shapes are symmetric we obtain a non-zero offset. In general, to reduce the Hall sensors offset, the current spinning technique is used, consisting in periodic commutation of current and voltage terminals [8].

Measurements regarding single phase offsets versus the biasing current were performed. For the basic and XL cells, this information is included in Figs. 3 and 4 respectively. The corresponding results were fitted in order to prove the parabolic behavior of the curves.

\section{B. Measurements of the Magnetic Equivalent Residual Offset Temperature Dependence}

The residual offset is an average of the four individual phases, as follows:

$$
V_{\text {residual(4phase) }}=\frac{V_{P_{1}}-V_{P_{2}}+V_{P_{3}}-V_{P_{4}}}{4}
$$

To obtain the magnetic field equivalent residual offset, $B_{\text {offset }}$ the following formula is used:

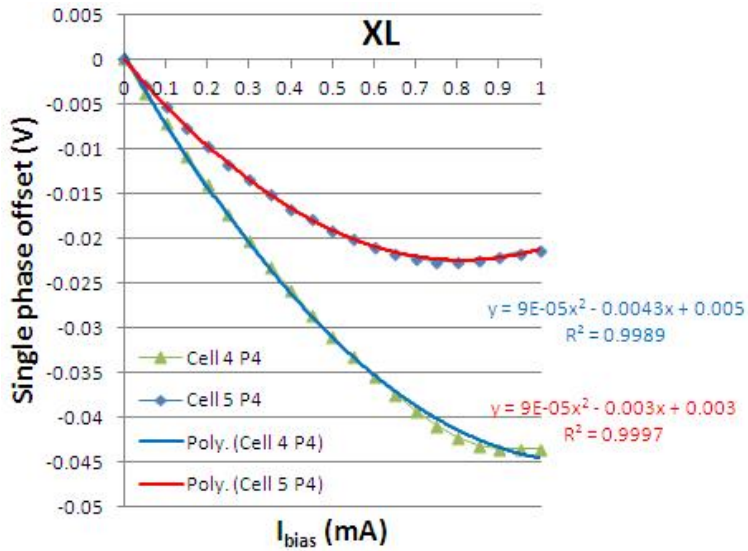

Fig. 4. Single phase offset (V) vs. $I_{\text {bias }}$ for XL cell.

$$
B_{\text {offset }}=\frac{V_{\text {residual(4phase })}}{S_{A}}
$$

where $S_{A}$ is the absolute sensitivity, according to Eq. 1 .

The measured magnetic equivalent residual offset of the Hall cells versus the temperature is presented for two currents, $I_{\text {bias }}=0.5 \mathrm{~mA}$, and $I_{\text {bias }}=1 \mathrm{~mA}$, in Figs. 5- 8. A similar type of graphs, but plotted against the absolute temperature was presented by the authors in [9]. We can observe that the XL Hall cell displayed the best behavior, by having the lowest offset and temperature drift.

When heating a sample, depending on the heating rate (fast or slow), the material behavior is different, due to the fact that the intimate physical processes are slightly different. In order to alleviate these embarrassing effects, we repeated the experiment for the same temperatures, with measurements taken at the natural (non-forced) cooling of the probe. Considering that these latter measurements are representative for the studied phenomenon, the measurements used in the statistical processing of data are the ones obtained at the natural cooling.

We had several temperature cycles. We started from room temperature, decreased the temperature to $0^{\circ}$ and then increased it to $125^{\circ}$, then decreased it again to $-40^{\circ}$ and then finally reverted to the room temperature. This is why on the previously presented figures we have several measurements for the same temperature point.

In order to calculate the offset drift, according to the work in papers [10], [11], a linear region between $-25^{\circ}$ and $-85^{\circ}$ was selected. We selected the above mentioned temperature interval on the natural cooling measurements region and performed a linear fit.

The offset drift is defined as the slope of the offset variation curve with temperature:

$$
V_{\text {offset }}=a T+b,
$$

where $a=\frac{d V_{\text {offset }}}{d T}$ is the offset drift.

Subsequently, the offset drift value presented in Tab. I is obtained by taking the slopes of the linear fittings on curves like the ones in Figs. 9 and 10. For the borderless cell, the offset drift is $0.6 \mu \mathrm{T} /{ }^{\circ} \mathrm{C}$, while the XL cell displayed among the tested cells, the lowest drift, $0.1 \mu T /{ }^{\circ} \mathrm{C}$. 


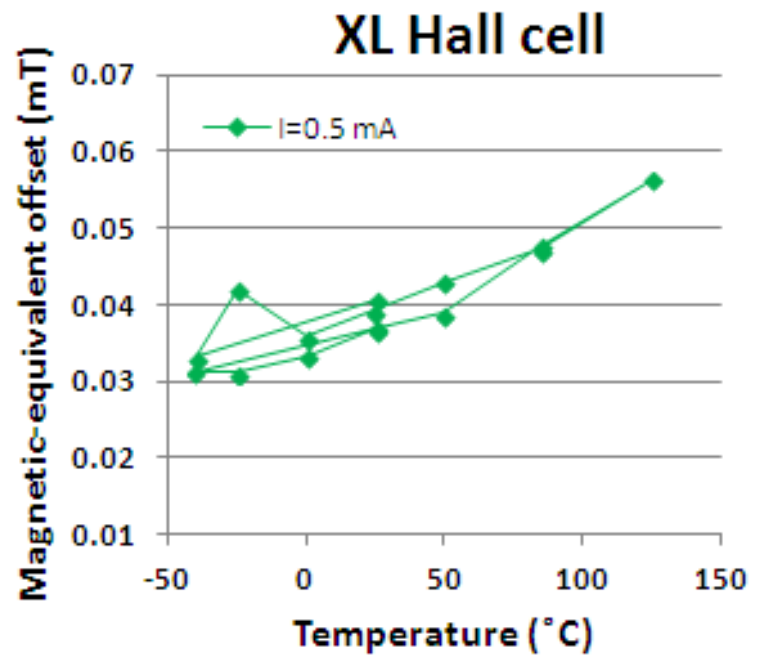

a)

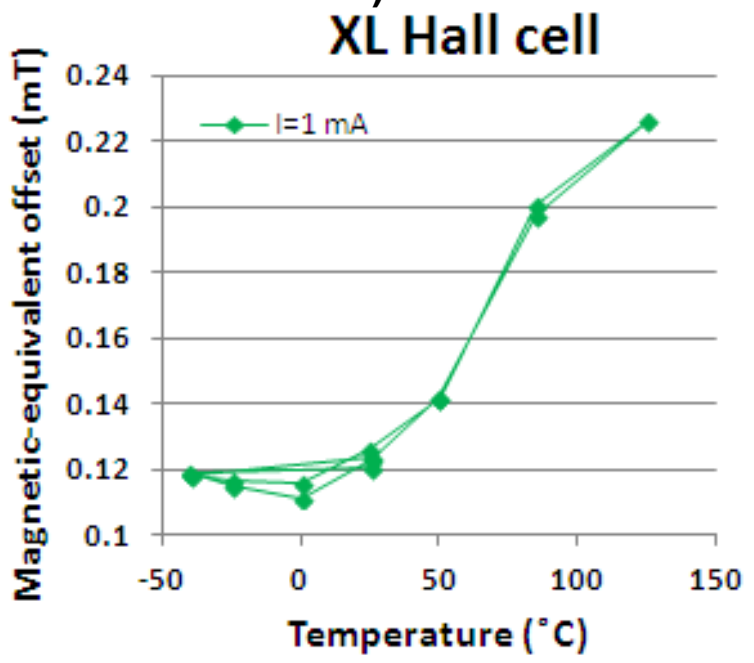

b)

Fig. 5. Magnetic equivalent residual offset for the XL Hall cell for $I_{\text {bias }}=$ $0.5 \mathrm{~mA}$ a) and $I_{\text {bias }}=1 \mathrm{~mA}$ b).

\section{Measurements of the Residual Offset vs. Biasing Current}

For the borderless cell, the measurements results concerning the residual offset vs. biasing current are displayed in Fig. 11. At this point, we have to mention that the residual offset was tested eight times for the same cell using the automated measurement system. Therefore, the residual offset presented in Fig. 11 is already averaged on 4 phases.

\section{Temperature Coefficient of Specific Quantities}

We were interested to see if the initial offsets, residual offset (both 2 and 4-phase) and resistance have the same temperature drift coefficient, information useful for developing future temperature correction blocks.

From measurements, the coefficient of variation with temperature was computed for the above-mentioned parameters and the data for three cells and two biasing currents is summarized in the Tabs. III and IV.

From the tables below, we can observe that for the resistance, the experimental results are in good agreement with the

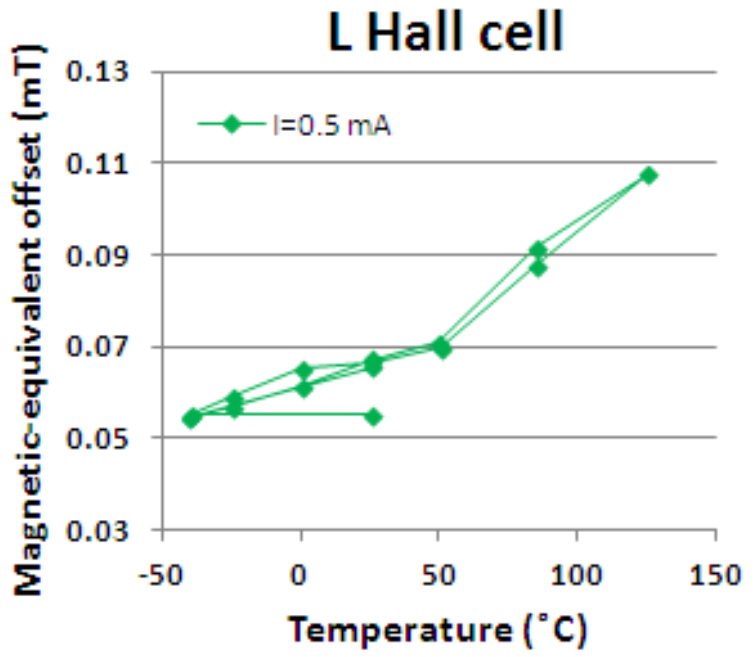

a)

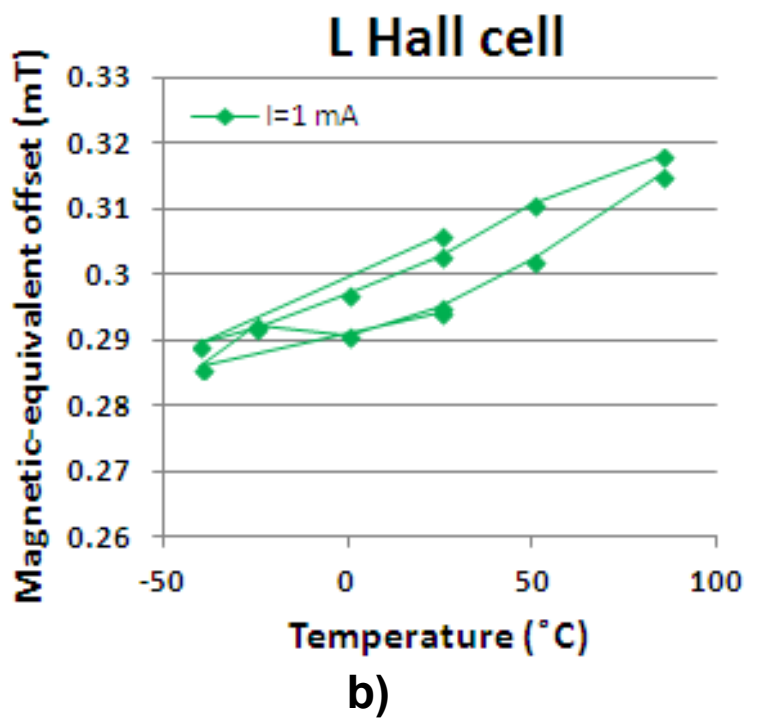

Fig. 6. Magnetic equivalent residual offset for the $\mathrm{L}$ Hall cell for $I_{\text {bias }}=$ $0.5 \mathrm{~mA}$ a) and $I_{\text {bias }}=1 \mathrm{~mA} \mathrm{~b}$ ).

value for provided by the technology used.

At low biasing current, the junction field effect is less, so we could expect a linear region in which the analyzed quantities to have a closer connection. At biasing current of $1.25 \mathrm{~mA}$, indeed the residual offset for the 2 phases (for basic and XL cells) has a higher variation with temperature, like the other analyses for 2-phase vs. 4-phase current spinning technique have shown before.

Among the four integrated and subsequently tested cells, XL cell displayed the minimum offset at room temperature, the lowest residual offset drift and the best sensitivity. So we can observe that this particular cell is the optimum one amongst the analyzed Hall effect sensors.

\section{E. Hall Cells Linearity Analysis}

An analysis of the linearity of some of the integrated Hall cells, in terms of resistance variation with the current has been performed. The following table summarizes the data regarding this linearity, denoted by $\alpha$ and calculated as the ratio of 


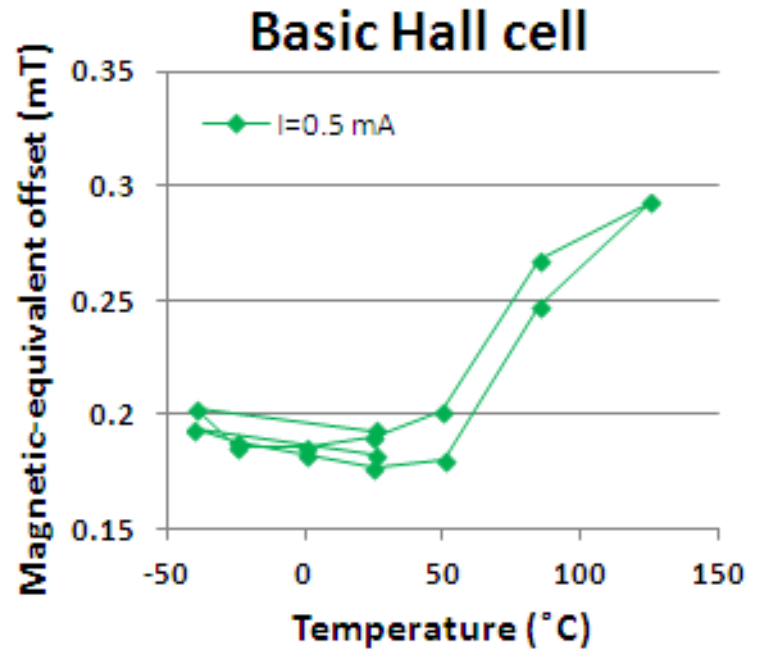

a)

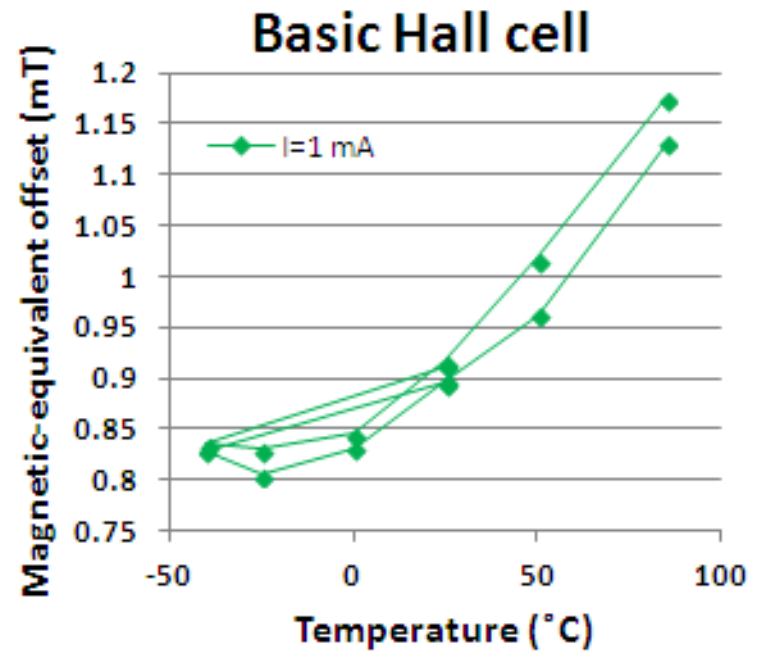

b)

Fig. 7. Magnetic equivalent residual offset for the basic Hall cell for $I_{b i a s}=$ $0.5 \mathrm{~mA}$ a) and $I_{\text {bias }}=1 \mathrm{~mA} \mathrm{~b}$ ).

the resistance for $S_{A}=0.03 \mathrm{~V} / T$ to the resistance for null absolute sensitivity. By consequence, $\alpha=\frac{R(0.03)}{R(0)}$. It is to be mentioned that $R(0)$ was not actually recorded for null absolute sensitivity, but for a very small experimental data, close to 0 .

Among the integrated cells, it seems that the closest linearity to unity is displayed by the cross-like cells.

\section{F. Three-dimensional Physical Simulations of the Hall Cells}

The TCAD Synopsys 3D physical simulator [12], based on numerical solutions to the carrier transport in semiconductors [13]-[15], proved to be a reliable tool to predict the Hall effect sensors performance, by investigating, amongst other parameters, their Hall voltage and sensitivity.

In Figs. 12 and 13, the Hall voltage (simulated values and measurement results) plotted against biasing current, for two different Hall cells, is presented. A good coherence between measurements and simulation results has been obtained, as you

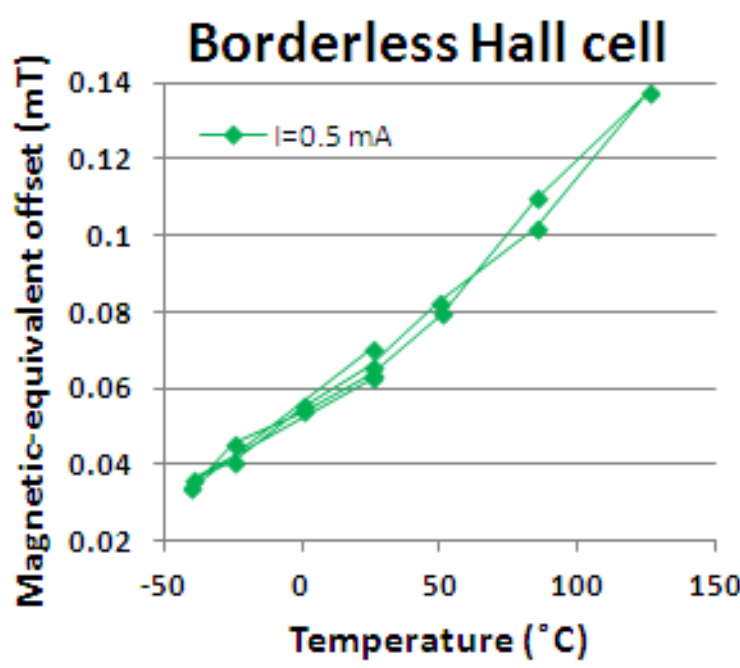

a)

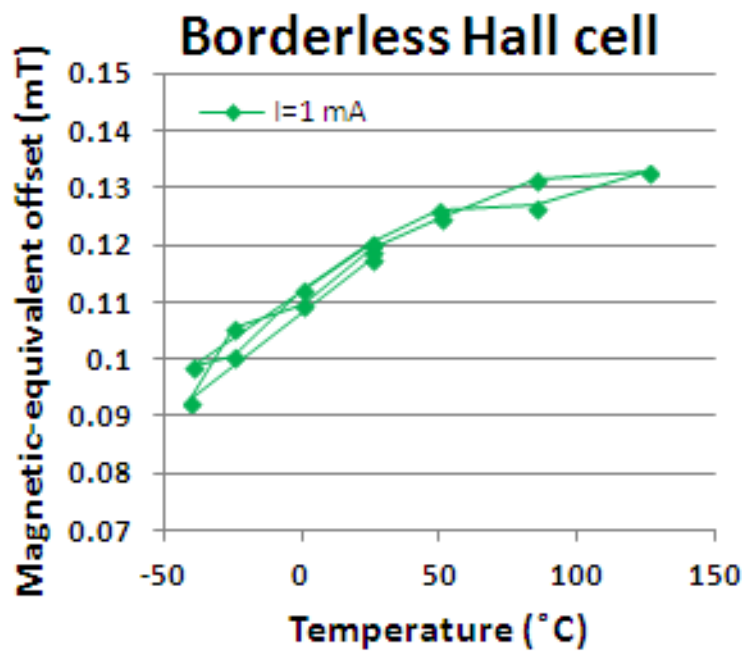

b)

Fig. 8. Magnetic equivalent residual offset for the borderless Hall cell for $I_{\text {bias }}=0.5 \mathrm{~mA}$ a) and $I_{\text {bias }}=1 \mathrm{~mA} \mathrm{~b}$ )

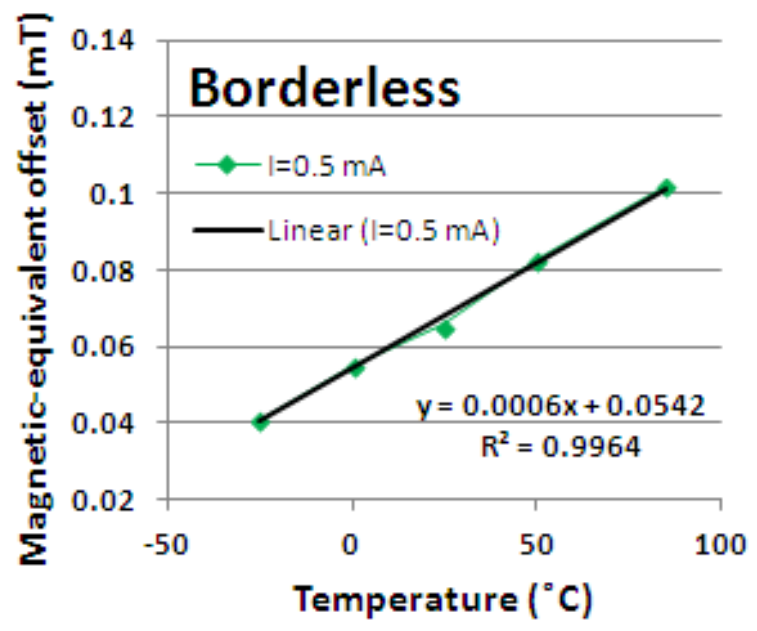

Fig. 9. Magnetic equivalent residual offset versus temperature for the borderless Hall cell. 


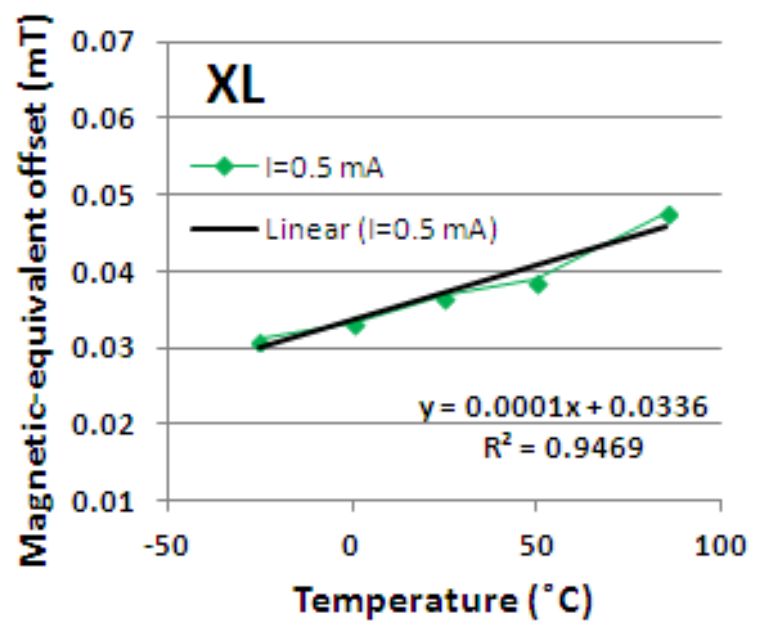

Fig. 10. Magnetic equivalent residual offset versus temperature for the XL Hall cell.

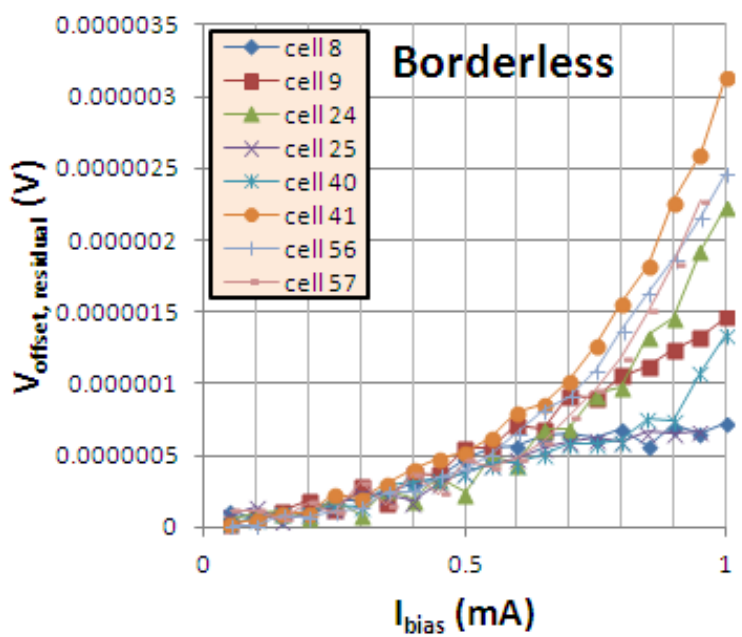

Fig. 11. 4-phase residual offset $(V)$ vs. $I_{\text {bias }}$ for borderless cell.

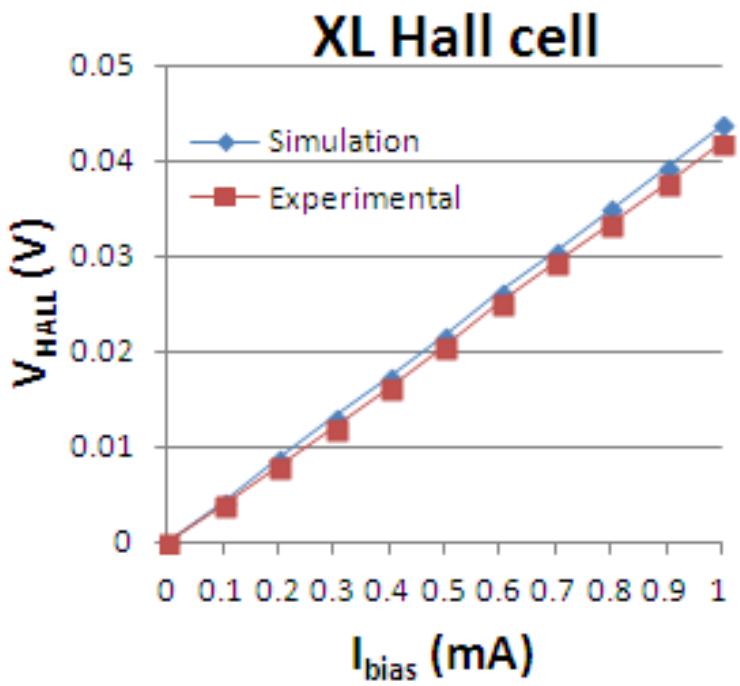

Fig. 12. Hall voltage (both simulated and experimental results) vs. biasing current, for the XL Hall cell.
TABLE III

TEMPERATURE COEFFICIENT FOR $I_{\text {bias }}=1.25 \mathrm{~mA}$

\begin{tabular}{|c|c|c|c|}
\hline $\begin{array}{c}\text { The } \\
\text { temperature } \\
\text { variation } \\
\text { coefficient }\end{array}$ & $\begin{array}{c}\alpha\left(T^{-1}\right) \\
\mathrm{X} 12-\mathrm{Basic} \\
R_{0}=2.37 k \Omega\end{array}$ & $\begin{array}{c}\alpha\left(T^{-1}\right) \\
\mathrm{X} 12-\mathrm{XL} \\
R_{0}=2.34 k \Omega\end{array}$ & $\begin{array}{c}\alpha\left(T^{-1}\right) \\
\mathrm{X} 12-\text { Borderless } \\
R_{0}=1.36 k \Omega\end{array}$ \\
\hline $\begin{array}{c}\text { Resistance } \\
(k \Omega)\end{array}$ & 0.004 & 0.004 & 0.0036 \\
\hline $\begin{array}{c}\text { Residual } \\
\text { offset (mV) } \\
4 \text { phases }\end{array}$ & 0.002 & 0.001 & 0.090 \\
\hline $\begin{array}{c}\text { Residual } \\
\text { offset (mV) } \\
2 \text { phases }\end{array}$ & -0.011 & 0.02 & 0.016 \\
\hline $\begin{array}{c}\text { Individual } \\
\text { offset (mV) } \\
\text { of phase P1 }\end{array}$ & -0.03 & -0.03 & 0.016 \\
\hline $\begin{array}{c}\text { Individual } \\
\text { offset (mV) } \\
\text { of phase P2 }\end{array}$ & -0.007 & -0.01 & 0.013 \\
\hline $\begin{array}{c}\text { Individual } \\
\text { offset (mV) } \\
\text { of phase P3 }\end{array}$ & -0.007 & -0.01 & 0.014 \\
\hline $\begin{array}{c}\text { Individual } \\
\text { offset (mV) } \\
\text { of phase P4 }\end{array}$ & -0.027 & & 0.0125 \\
\hline
\end{tabular}

TABLE IV

TEMPERATURE COEFFICIENT FOR $I_{\text {bias }}=0.25 \mathrm{~mA}$

\begin{tabular}{|c|c|c|c|}
\hline $\begin{array}{c}\text { The } \\
\text { temperature } \\
\text { variation } \\
\text { coefficient }\end{array}$ & $\begin{array}{c}\alpha\left(T^{-1}\right) \\
\text { X12-Basic } \\
R_{0}=2.37 k \Omega\end{array}$ & $\begin{array}{c}\alpha\left(T^{-1}\right) \\
\text { X12-XL } \\
R_{0}=2.34 k \Omega\end{array}$ & $\begin{array}{c}\alpha\left(T^{-1}\right) \\
\text { X12-Borderless } \\
R_{0}=1.36 k \Omega\end{array}$ \\
\hline $\begin{array}{c}\text { Resistance } \\
(k \Omega)\end{array}$ & 0.004 & 0.004 & 0.004 \\
\hline $\begin{array}{c}\text { Residual } \\
\text { offset (mV) } \\
4 \text { phases }\end{array}$ & 0.025 & 0.006 & 0.031 \\
\hline $\begin{array}{c}\text { Residual } \\
\text { offset (mV) } \\
2 \text { phases }\end{array}$ & 0.011 & 0.011 & -0.028 \\
\hline $\begin{array}{c}\text { Individual } \\
\text { offset (mV) } \\
\text { of phase P1 }\end{array}$ & -0.012 & -0.0009 & 0.031 \\
\hline $\begin{array}{c}\text { Individual } \\
\text { offset (mV) } \\
\text { of phase P2 }\end{array}$ & -0.008 & 0.002 & 0.025 \\
\hline $\begin{array}{c}\text { Individual } \\
\text { offset (mV) } \\
\text { of phase P3 }\end{array}$ & -0.008 & 0.002 & 0.013 \\
\hline $\begin{array}{c}\text { Individual } \\
\text { offset (mV) } \\
\text { of phase P4 }\end{array}$ & -0.0123 & 001 & 0.015 \\
\hline
\end{tabular}

can see in the following graphs, for two of the analyzed cells, namely XL and borderless cells respectively.

\section{CONCLUSIONS}

Different Hall effect sensors were integrated in a CMOS technology and their performance evaluated. Four geometries (basic, L, XL and borderless cells) have been chosen and tested. The offset, its drift and the sensitivity are quantities that were measured to determine the sensors performance. 
TABLE V HALl CELLS LINEARITY

\begin{tabular}{|c|c|c|}
\hline $\begin{array}{c}\text { Type of } \\
\text { Hall Cell }\end{array}$ & $\begin{array}{c}I_{\text {bias }}(m A) \text { for } \\
S_{A}=0.03 V / T\end{array}$ & $\alpha=R(0.03) / R(0)$ \\
\hline Basic & 0.4 & 1.022 \\
\hline L & 0.4 & 1.021 \\
\hline XL & 0.4 & 1.021 \\
\hline $\begin{array}{c}\text { Border- } \\
\text { less }\end{array}$ & 0.95 & 1.033 \\
\hline
\end{tabular}

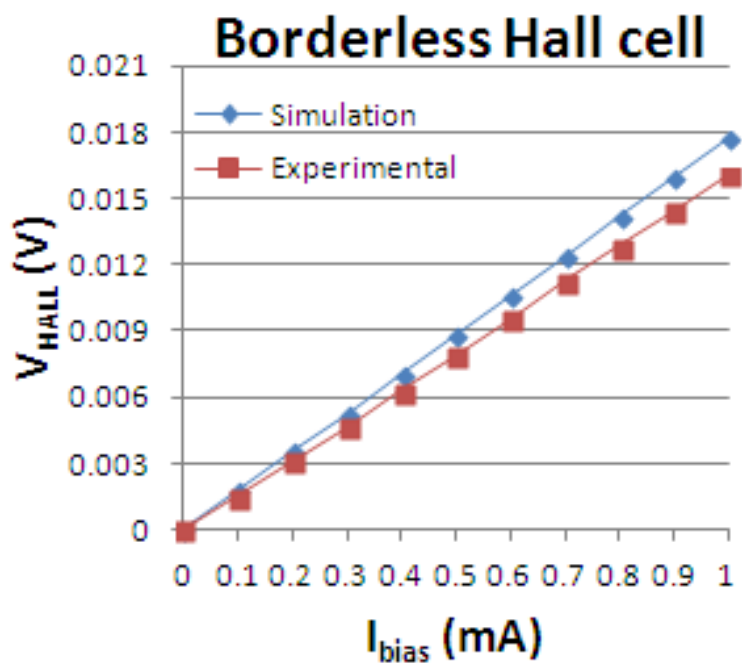

Fig. 13. Hall voltage (both simulated and experimental results) vs. biasing current, for the borderless Hall cell.

The results corresponding to the residual offset and its behavior with the temperature were presented, with a comparative analysis on different Hall cell types. The temperature coefficient of the individual offset, residual offset (using 2phase and 4-phase current spinning technique) and resistance has been computed for several sensors. This information might be useful in designing future temperature correction blocks.

It was shown that there is coherence between the measurement and the simulation results. We finally concluded which of the integrated shapes exhibited the best performance.
The various shapes analyzed offer an overview on how the performance is related to the geometry.

As future work, we aim at investigating the residual offset prediction and spinning current technique efficiency by using the 3D physical simulator.

\section{REFERENCES}

[1] E. Ramsden, Hall-Effect Sensors - Theory and Applications, 2nd ed. Elsevier, 2006.

[2] S.-Y. Kim, C. Choi, K. Lee, and W. Lee, "An improved rotor position estimation with vector-tracking observer in PMSM drives with lowresolution Hall-effect sensors," IEEE Transactions on Industrial Electronics, vol. 58, no. 9, pp. 4078-4086, 2011.

[3] M. A. Paun, J. M. Sallese, and M. Kayal, "Geometry influence on Hall effect devices performance," U.P.B. Sci. Bull., vol. 72, no. 4, pp. 257271, 2010.

[4] - , "Hall effect sensors performance investigation using threedimensional simulations," P. of the 18th International Conference, Ed. Mixed Design of Integrated Circuits and Systems (MIXDES), 2011, pp. $450-455$.

[5] — "Comparative study on the performance of five different Hall effect devices," Sensors, vol. 13, no. 2, pp. 2093-2112, 2013.

[6] _ "Geometrical parameters influence on the Hall effect sensors offset and drift," in 7th Conference on Ph.D. Research in Microelectronics and Electronics (PRIME), 2011, pp. 145-148.

[7] R. S. Popovic, Hall Effect Devices, 2nd ed. Institute of Physics Publising, 2004.

[8] Y. Hu and W.-R. Yang, "CMOS hall sensor using dynamic quadrature offset cancellation," in Solid-State and Integrated Circuit Technology, 2006, ICSICT '06. 8th International Conference on, 2006, pp. 284 286.

[9] M. A. Paun, J. M. Sallese, and M. Kayal, "Hall effect sensors design, integration and behaviour analysis," Journal of Sensors and Actuator Networks, vol. 2, no. 1, pp. 85-97, 2013.

[10] H. Blanchard, C. D. Iseli, and R. S. Popovic, "Compensation of the the temperature-dependent offset drift of a Hall sensor," SENSORS AND ACTUATORS A-PHYSICAL, vol. 60, no. 1-3, pp. 10-13, 1997.

[11] H. Blanchard, F. De Montmollin, and J. Hubin, "Highly sensitive Hall sensor in CMOS technology," SENSORS AND ACTUATORS APHYSICAL, vol. 82, no. 1-3, pp. 144-148, 2000.

[12] "Synopsys TCAD tools," 2013. [Online]. Available: http://www.synopsys.com/Tools/TCAD

[13] S. M. Sze and K. K. Ng, Physics of semiconductor devices, 3rd ed John Wiley and Sons, 2007.

[14] I. S. Selberherr, Analysis and Simulation of Semiconductor Device. Springer-Verlag, 1984.

[15] W. Allegretto, A. Nathan, and H. Baltes, Numerical Analysis of Magnetic-Field-Sensitive Bipolar Devices. IEEE Transactions On Computer-Aided Design, 1991, vol. 10, no. 4. 